\title{
A simplified strategy to package foreign proteins into baculovirus occlusion bodies without engineering the viral genome
}

\author{
María Laura Fabre, Tomás Masson, Santiago Haase ${ }^{1}$, María Leticia Ferrelli, Víctor Romanowski * \\ Instituto de Biotecnología y Biología Molecular (IBBM, UNLP-CONICET), Facultad de Ciencias Exactas, Universidad Nacional de La Plata, La Plata, Buenos Aires, Argentina
}

\section{A R T I C LE IN F O}

Keywords

AgMNPV

AcMNPV

Polyhedron envelope protein (PEP)

UFLAg-286

\begin{abstract}
A B S T R A C T
Polyhedron envelope protein (PEP) is the major component of the calyx that surrounds the baculovirus occlusion body (OB). PEP has been associated with the stabilization and resistance of polyhedra in the environment. Due to the abundant levels of PEP in OBs, we decided to use this protein as a fusion partner to redirect foreign proteins to baculovirus polyhedra. In this study we developed a strategy that involves the generation of a monoclonal transformed insect cell line expressing a protein of interest fused to the the Anticarsia gemmatalis multiple nucleopolyhedrovirus (AgMNPV) N-terminus of PEP that enables the packaging of foreign proteins into the OBs without generating a recombinant baculovirus. This proved to be an efficient platform that could be exploited to improve wild type baculovirus for their use as bioinsecticides without facing the concerns of releasing genetically modified DNA to the environment and bypassing the associated regulatory issues. We demonstrated, using immunological, proteomic and microscopy techniques, that the envelope of AgMNPV OBs can effectively trap chimeric proteins in an infected insect cell line expressing AgMNPV PEP fused to the enhanced green fluorescent protein (eGFP). Furthermore, packaging of chimeric PEP also took place with heterologous OBs such as those of Autographa californica multiple nucleopolyhedrovirus (AcMNPV), another group I alphabaculovirus.
\end{abstract}

\section{Introduction}

The family Baculoviridae consists of rod-shaped, enveloped, occluded viruses that contain circular double-stranded DNA genomes of 80-180 kb and infect insects from the orders Lepidoptera, Diptera and Hymenoptera (Haase et al., 2013; Harrison et al., 2018). In the environment, mainly on the foliage and in the soil, baculoviruses are found as occlusion bodies (OBs) which contain one or more enveloped nucleocapsids embedded in an abundant matrix composed mainly of a protein designated polyhedrin in nucleopolyhedrovirus (NPVs) or granulin in granulovirus (Gvs) (Jehle et al., 2006). The OB are surrounded by a calyx or polyhedron envelope (PE), composed mainly of polyhedron envelope protein (PEP) (Rohrmann, 2013). Insects feeding on contaminated foliage ingest the OBs. Once in the midgut, the OBs are dissolved, releasing the occlusion-derived virions (ODVs) that enter the epithelial cells to initiate an infectious cycle. Early in infection budded virions (BVs) are produced and spread throughout the permissive tissues of the insect. At late stages of infection a different type of enveloped virions accumulate and become occluded to form OBs within the nuclei of NPV-infected cells (Rohrmann, 2013). In view of their pathogenic potential, baculoviruses have been studied as promis- ing bioinsecticides and over three decades several commercial products became available (Haase et al., 2015; Kroemer et al., 2015; López et al., 2018,). Baculoviruses are especially attractive biological control agents due to their narrow host range and the long viability of their OBs that can persist in a hostile environment. However, they exhibit some limitations such as the slow speed of kill compared to chemical quick action pesticides.

When OBs reach the larval midgut, released ODVs must cross the first barrier consisting of the peritrophic membrane (PM) a layer separating the midgut lumen form the epithelial cells. Some baculoviruses have been engineered to improve their infectivity, embedding enhancing factors into the OBs to increase the permeability of the PM (Hegedus et al., 2009; Mitsuhashi et al., 2007; Yang et al., 2017). To achieve packaging of foreign insecticidal proteins into genetically modified OBs fusion to a second copy of polyhedrin (POLH) has been extensively explored (Ali et al., 2015; Chen et al., 2013; López et al., 2018,). Another OB target protein not fully investigated thus far is the polyhedron envelope protein (PEP), also known as pp34 or calyx, a $34.5 \mathrm{kDa}$ virus-specific phosphoprotein that forms multiple layers surrounding the OBs. Sajjan and Hinchigeri (2016) reported that mature PEP layers represent approximately one-third of the BmNPV

\footnotetext{
* Corresponding author.

E-mail address: victor@biol.unlp.edu.ar (V. Romanowski)

1 Present address: Department of Cell \& Developmental Biology, The University of Michigan School of Medicine, MSRB II, RM 4570C, 1150 West Medical Center Drive, Ann Arbor, MI 48109-5689, USA.
} 
OB volume. Recently, a truncated version of PEP was engineered to display nano-ZnO particles (Li et al., 2015). This modification improved the UV resistance of the recombinant Autographa californica multiple nucleopolyhedrovirus (AcMNPV) OBs. The authors of that study did not observe complementation between PEP of different baculoviruses.

In view of the high abundance of PEP, we considered this protein as an interesting target to drive foreign selected polypeptides to the outer layer of the OB. This localization would allow a more immediate and direct interaction with the PM barrier during the peroral infection of susceptible larvae. We chose Anticarsia gemmatalis multiple NPV (AgMNPV) as a model due to the success of this baculovirus used to control the populations of the velvet bean caterpillar, Anticarsia gemmatalis (Lepidoptera: Noctuidae), an important defoliator of soybean fields in warm territories of South America (Levy et al., 2011; Moscardi, 1999). However, its performance is much lower in temperate climates and efforts must be addressed to improve its speed of action in other areas of the Americas.

The study aimed to develop a stably transformed insect cell line as a platform for delivery of recombinant proteins into OBs of wild type baculovirus as a way to improve its insecticidal properties. We present a stably transformed UFLAg insect cell line that constitutively expresses the fusion polypeptide of GFP and PEP from AgMNPV and test the expression and localization of the chimeric protein in the wild type OBs of two baculoviruses AgMNPV (narrow host range) and AcMNPV (wide host range).

\section{Materials and methods}

\subsection{Cells and viruses}

The UFLAg-286 cell line was grown at $28^{\circ} \mathrm{C}$ in Grace's (Invitrogen $^{\mathrm{TM}}$ ) medium containing $10 \%$ fetal bovine serum (Internegocios S.A., Mercedes, Argentina) (Sieburth and Maruniak, 1988). AgMNPV-2D and AcMNPV-C6 isolates were propagated in UFLAg-286 and High Five (BTI-TN-5B1-4) cells, respectively. Infection assays followed the standard procedures (O'Reilly et al., 1994).

\subsection{Construction of the expression vector}

The expression plasmid pIP-V5/His is a modified version of the pIB-V5/His (Invitrogen ${ }^{\mathrm{TM}}$ ) in which the blasticidin resistance gene was replaced with the puromycin selection cassette. This plasmid contains a constitutive early promoter (pOie2) and a polyadenylation signal derived from the ie1 gene of Orgyia pseudotsugata MNPV. To generate the pIP-GFP::PEP ${ }_{\text {Ag }}$ plasmid, the ORF encoding for PEP was amplified from AgMNPV-2D (Genbank accession number NC_008520) by PCR with primers Fpep (5' GAGCTCACTGGATCCGGCGGAGGCGGAAGCTACGCTGTACCTACAATATCATT $3^{\prime}$ ) and Rpep (5'GACTCTCGAGTTATTTGCGACTGCCCATC3') harboring restriction endonucleases sites (underlined) along with a linker sequence (coding for peptide GGGGS, in bold). PCR conditions involved 30 cycles of $94^{\circ} \mathrm{C}$ for $45 \mathrm{~s}, 55^{\circ} \mathrm{C}$ for $20 \mathrm{~s}$, and $72^{\circ} \mathrm{C}$ for $45 \mathrm{~s}$. PCR product was cloned into pGEM-T Easy ${ }^{\mathrm{TM}}$ (Invitrogen $^{\mathrm{TM}}$ ) and then excised by digestion with SacI and XhoI enzymes. This fragment was then inserted between SacI-XhoI sites in pIP-V5/His vector to generate the PIP-PEP $\mathrm{Ag}$. The egfp reporter gene (coding for the enhanced green fluorescent protein eGFP, hereinafter referred to as GFP) was amplified from the pEGFP-N3 plasmid (Clontech) by PCR using Fegfp (5' GGTACCACTGAGCTCAGATCTATGGTGAGCAAGGGCGAG 3') and Regfp (5' GGATCCTCTAGATGACTTGTACAGCTCGTCCATGCC 3') primers. PCR conditions were those described above. The resulting egfp fragment was inserted into the PGEM-T Easy ${ }^{\mathrm{TM}}$ and then sub-cloned in the pIP-PEP ${ }_{\mathrm{Ag}}$ between SacI and BamHI sites and also in the pIP-V5/ His (SacI-BamHI) to generate the control plasmid pIP-GFP. All plasmids containing PCR-derived frag- ments were confirmed by Sanger sequencing (Macrogen Corporation, South Korea).

\subsection{Cell lines constitutively expressing GFP and GFP::PEP $A g$}

UFLAg-286 cells were seeded in a 6-well plate $\left(2 \times 10^{6}\right.$ cells/well $)$ and transfected with $1 \mu \mathrm{g}$ of plasmid DNA using $3 \mu \mathrm{lof} 1 \mathrm{mg} / \mathrm{ml}$ polyethyleneimine (PEI) according to Ogay et al. (2006) to generate the UFLAg-GFP::PEPAg and UFLAg-GFP cell lines. Briefly, $1 \mu \mathrm{g}$ of the plasmid DNA was mixed with $100 \mu \mathrm{L}$ of $150 \mathrm{mM} \mathrm{NaCl}$ and mixed gently with PEI. After incubation at room temperature for 5 min DNA-PEI complex was added to the cells. At $24 \mathrm{~h}$ post transfection, the medium was replaced with fresh medium containing $10 \mu \mathrm{g} / \mathrm{mL}$ puromycin and incubated for 2-3 weeks with occasional replacements. Clones of puromycin-resistant cells were isolated using the terminal dilution method (Lynn and Harrison, 2016). Wells containing cells were examined by fluorescence microscopy using a Nikon eclipse $t i$ with a GFP filter. The same clones of UFLAg-GFP and UFLAg-GFP::PEP ${ }_{\text {Ag }}$ were used for all subsequent experiments in parallel with the original UFLAg-wt cell line.

\subsection{Occlusion bodies purification}

UFLAg-wt, UFLAg-GFP, and UFLAg-GFP::PEP $\mathrm{Ag}_{\mathrm{Ag}}$ cell lines $\left(10^{7}\right.$ cells each) were infected with AcMNPV and AgMNPV (MOI: 10 pfu/cell). At $120 \mathrm{~h}$ p.i., the polyhedra were purified according to a standard protocol (O'Reilly et al., 1994). Briefly, the cells were sedimented at 3000 $\mathrm{x} \mathrm{g}$ for $5 \mathrm{~min}$, resuspended in $\mathrm{ddH}_{2} \mathrm{O}$ and sonicated for two cycles of $30 \mathrm{~s}$ at $50 \%$ potency (Sonics Vibra-Cell) in ice. Cell debris was eliminated by centrifugation ( $500 \mathrm{x} \mathrm{g}, 2 \mathrm{~min}$ ) and supernatant with viral OBs was centrifuged at $15,000 \times g$ for $20 \mathrm{~min}$. OBs were washed twice and resuspended in PBS. OBs were loaded onto $50 \%(w / w)$ sucrose cushion and centrifuged at $15,000 \times g 20 \mathrm{~min}$ at $4^{\circ} \mathrm{C}$ in a microcentrifuge (Eppendorf). The pellet containing the OBs was washed twice and resuspended in PBS. Finally, OBs were counted using Neubauer's hemocytometer mounted on a phase-contrast light microscope with a $20 \mathrm{x}$ objective. GFP-positive OBs in each sample were detected with a fluorescence microscope (40x objective). Three images containing more than 1000 OBs each were counted for all the samples and analyzed with ImageJ software, (https://imagej.nih.gov/ij/). The results were plotted in the form of a bar graph: \% GFP-positive OBs $v$ s. total number of OBs in samples of AgMNPV and AcMNPV.

\subsection{Confocal microscopy and SEM}

AcMNPV and AgMNPV OBs purified from infected UFLAg-GFP, and UFLAg-GFP::PEP $\mathrm{Ag}_{\mathrm{Ag}}$ cells in independent experiments were visualized in a Leica TCS SP5 confocal laser scanning microscope (63x optical zoom). Scanning electron microscopy (SEM) was used to examine the polyhedron envelope on the surface of OBs derived from UFLAg-GFP::PEP ${ }_{\mathrm{Ag}}$ cells infected AgMNPV and AcMNPV. OB samples were fixed overnight in suspension by mixing with an equal volume of fixative ( $4 \%$ formaldehyde and $1 \%$ glutaraldehyde in $0.1 \mathrm{M}$ phosphate buffer, $\mathrm{pH} 7.4$ ) and subsequently washed twice with $0.1 \mathrm{M}$ phosphate buffer. Samples were then partially dehydrated with $70 \%$ ethanol, dried, placed on aluminum mounts using carbon tags, sputter-coated with gold-palladium and photographed (magnification, $10000 \times ; 12,5 \mathrm{kV}$, FEI Quanta 200). Fifteen OBs of each virus were selected randomly and size measured to calculate the average diameter.

\subsection{Western blot}

Cell pellets from infected and uninfected UFLAg-wt, UFLAg-GFP and UFLAg-GFP::PEP $\mathrm{Ag}$ and negative and positive control samples 
(wt UFLAg-286 cells and purified GFP protein, respectively) were washed with PBS and resuspended in RIPA buffer containing 1:100 protease inhibitor cocktail (Sigma-Aldrich). The protein extracts were incubated in sample buffer containing a protease inhibitor cocktail (Sigma-Aldrich) and heated at $100 \mathrm{C}$ for $5 \mathrm{~min}$. Samples were separated by $10 \%$ SDS-PAGE and transferred onto a PVDF membrane by wet electrophoresis. The membranes were blocked with $5 \%$ non fat milk in Tris-buffered saline (TBS) containing $0.01 \%$ Tween 20 and were incubated with an anti-GFP monoclonal antibody (Chromotek, 3\#9) for $1 \mathrm{~h}$ at room temperature, washed three times with TBS-Tween, and incubated with horseradish peroxidase (HRP)-coupled anti-rat IgG (Thermo Fisher Scientific, \# 61-9520) at room temperature for $1 \mathrm{~h}$. The membrane was washed three times with TBS-Tween and developed with ECL Reagent (Thermo Fisher Scientific).

\subsection{Mass spectrometry analysis}

AcMNPV and AgMNPV OBs samples derived from UFLAg-GFP::PEP $\mathrm{Ag}_{\mathrm{Ag}}$ cells were quantified by Bradford assay (Bradford, 1976) and protein samples were treated as described in Masson et al. (2019). Analyses were performed at the Proteomics Core Facility CEQUIBIEM, UBA-CONICET (University of Buenos Aires - National Research Council). The digested samples were analyzed by nano LC-MS/MS in a Thermo Scientific Q Exactive Mass Spectrometer coupled to a nano-HPLC EASY-nLC 1000 (Thermo Scientific). A voltage of $3.5 \mathrm{kV}$ was used for ElectroSpray Ionization (Thermo Scientific, EASY- SPRAY). Full-scan mass spectra were acquired in the Orbitrap analyzer. The scanned mass range was $400-1800 \mathrm{~m} / \mathrm{z}$, at a resolution of 70,000 at $400 \mathrm{~m} / \mathrm{z}$ and the $12 \mathrm{most}$ intense ions in each cycle, were sequentially isolated, fragmented by higher-energy collisional dissociation (HCD). Peptides with a charge of +1 or with unassigned charge state were excluded from fragmentation for MS2.

\subsection{MS data analysis}

Q Exactive raw data was analyzed using Proteome Discoverer ${ }^{\mathrm{TM}}$ software (version 2.1.1.21, Thermo Scientific) and searched against AgMNPV and AcMNPV proteomes database (accession numbers DQ813662. 2 and KM667940, National Center for Biotechnology Information www. ncbi.nlm.nih.gov) digested in silico with trypsin with a maximum of one missed cleavage per peptide. A $10 \mathrm{ppm}$ was set as precursor mass tolerance and a $0.05 \mathrm{Da}$ as product ion tolerance. Protein hits were filtered for high confidence peptide matches with a maximum protein and peptide false discovery rate of $1 \%$ calculated using a reverse database strategy. LC-MS/MS data were analyzed by Proteome Discoverer ${ }^{\mathrm{TM}}$ software for a label-free protein quantification using a the emPAI values. EmPAI values of GFP:: EEP $_{\mathrm{Ag}}$ were converted to molar percentages by normalizing against the sum of all emPAI values (Shinoda et al., 2009). Molar percentage (mol \%): emPAI GFP::PEPAg $/ \Sigma(\mathrm{emPAI}) * 100$.

\section{Results}

\subsection{Development of stably transformed insect cell lines}

UFLAg-286 cells were engineered to constitutively express the

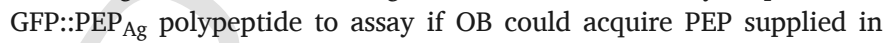
trans during morphogenesis. To this end, two expression plasmids were constructed: pIP-GFP:: PEP $_{\mathrm{Ag}}$ containing the complete sequence of AgMNPV PEP, hereafter designated $\mathrm{PEP}_{\mathrm{Ag}}$, fused at its $\mathrm{N}$-terminus to the enhanced green fluorescent protein (GFP), and pIP-GFP containing the egfp gene ORF as a control plasmid (Fig. 1a). These plasmids were used to develop two stably transformed insect cell lines: UFLAg-GFP::PEP $\mathrm{Ag}_{\mathrm{Ag}}$, expressing the GFP::PEP ${ }_{\mathrm{Ag}}$ polypeptide and UFLAg-GFP, generated

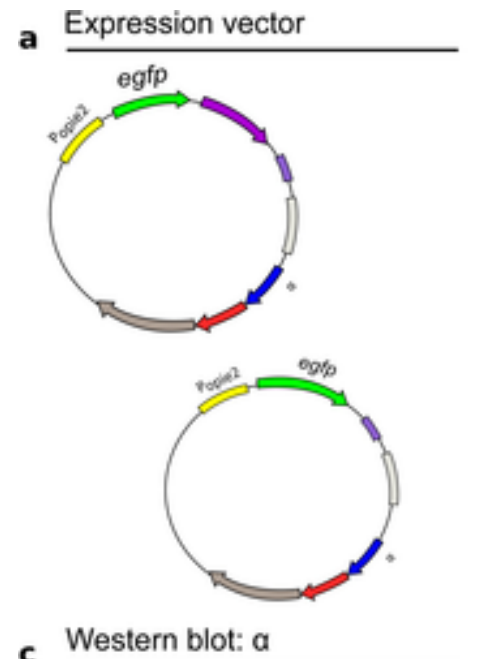

b Fluorescence microscopy
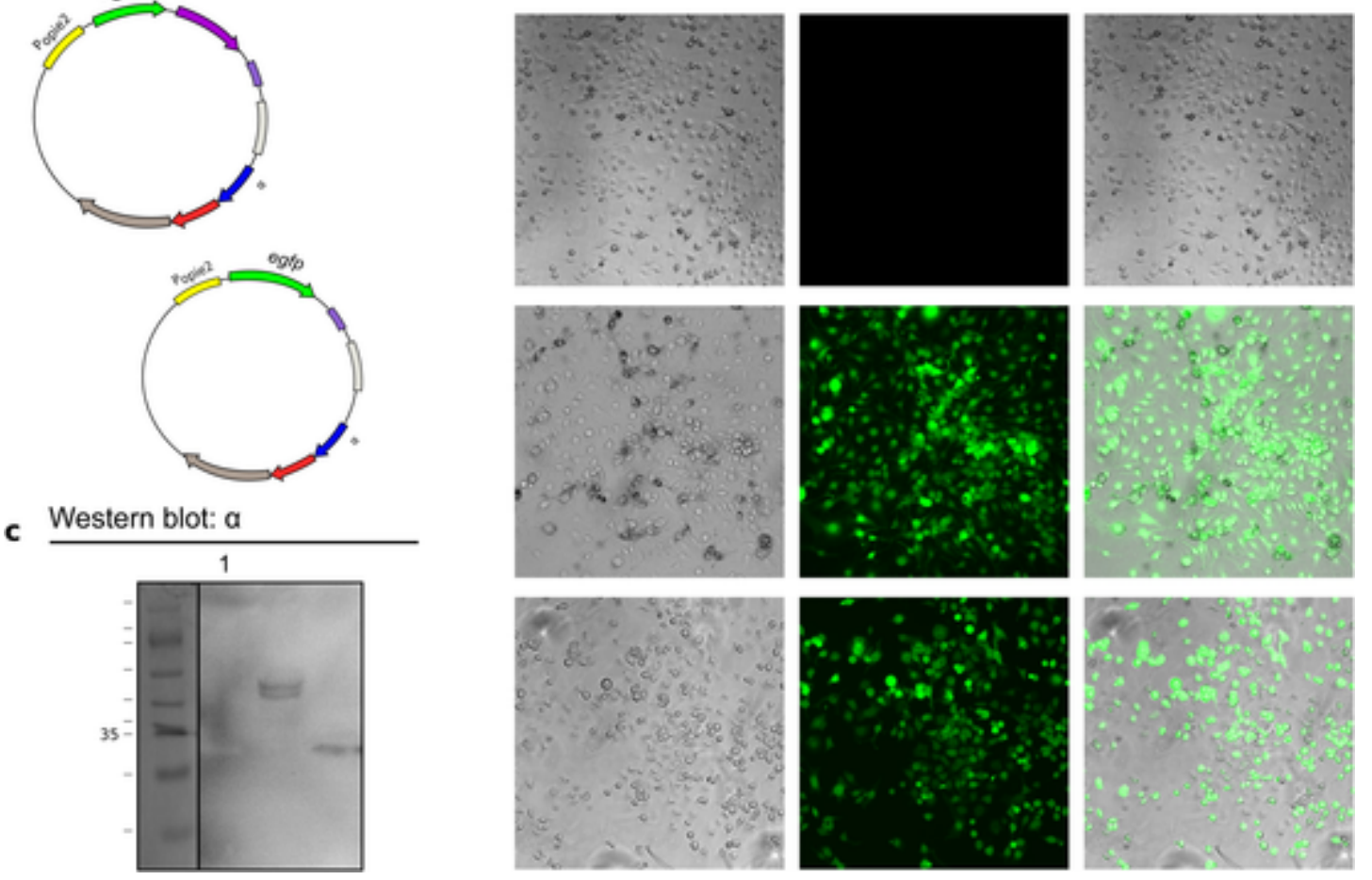

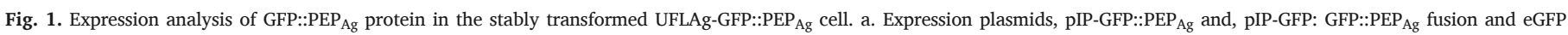

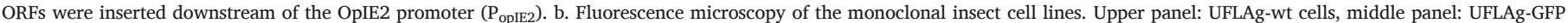

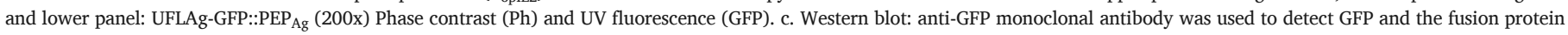

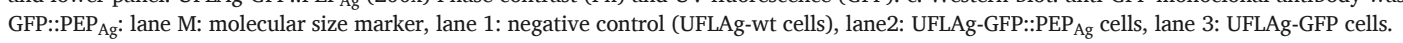


to test if GFP alone could be incorporated into the OBs. UFLAg-286 cells were transfected with the corresponding expression plasmids and then incubated with selection medium until several puromycin resistant clones were obtained. The presence of the eGFP reporter gene in both transformed cells was assessed by fluorescence microscopy, confirming the successful production of both recombinant proteins, GFP::PEP $\mathrm{Ag}_{\mathrm{Ag}}$ and eGFP, in the selected cell lines (Fig. 1b). Western Blot analysis confirmed the expression of GFP::PEP ${ }_{\mathrm{Ag}}$ in UFLAg-GFP::PEP $\mathrm{Ag}_{\mathrm{Ag}}$ cells. A $65 \mathrm{kDa}$ band was observed corresponding to the expected size for the GFP:: PEP $_{\text {Ag }}$ fusion product (Fig. 1c). UFLAg-286 were used as negative control and UFLAg-GFP cells as positive control for GFP expression.

\subsection{Targeting of GFP::PEP ${ }_{A g}$ to AgMNPV and AcMNPV OBs}

To evaluate the localization of the GFP:: $\mathrm{PEP}_{\mathrm{Ag}}$ protein in the $\mathrm{OBs}$, UFLAg-GFP::PEP ${ }_{A g}$ cells were infected with wild type AgMNPV. As previously reported by Sieburth and Maruniak (1988), UFLAg-286 cells are also susceptible to AcMNPV; therefore, UFLAg-GFP::PEP $\mathrm{Pg}_{\mathrm{Ag}}$ were also used to analyze if GFP:: $\mathrm{PEP}_{\mathrm{Ag}}$ could be included in wild type AcMNPV OBs. Light microscopy analysis showed that constitutive expression of the recombinant protein in UFLAg-GFP::PEP $\mathrm{Ag}_{\mathrm{Ag}}$ did not affect the replication of AgMNPV and AcMNPV, and produced well-formed polyhedra (Fig. 2a). Same results were obtained with the control cell line UFLAg-GFP, infected with AgMNPV or AcMNPV (Fig. 2b). Purified OBs of AgMNPV or AcMNPV obtained by infection of cell lines UFLAg-wt, UFLAg-GFP and UFLAg-GFP::PEP ${ }_{\mathrm{Ag}}$ in parallel experiments, were analyzed by western blot with anti-GFP antibody to confirm the localization of the fusion protein and to corroborate if $\mathrm{PEP}_{\mathrm{Ag}}$ could drive the inclusion of GFP into the OB (Fig. 3a). The OBs derived from UFLAg-GFP::PEP ${ }_{A g}$ infected cells were GFP positive, showing the corresponding polypeptide band size $(65 \mathrm{kDa})$. On the other hand, there was no GFP positive signal in OBs derived from UFLAg-GFP infected cells confirming that GFP alone did not drive inclusion into the OBs. These results demonstrated that $\mathrm{PEP}_{\mathrm{Ag}}$ is the only responsible for the localization of GFP::PEP ${ }_{\mathrm{Ag}}$ in OBs of AgMNPV and AcMNPV OBs. Even though, pep gene products have been described to be virus-species-specific, the experiments indicated that $\mathrm{PEP}_{\mathrm{Ag}}$ can also be included in the heterologous AcMNPV OBs in presence of the wt PEP encoded by this virus.

\subsection{Packaged OBs analyzed by microscopy}

Confocal microscopy analysis of AgMNPV and AcMNPV OBs produced in UFLAg-GFP:: PEP $_{\mathrm{Ag}}$ infected cells confirmed the presence

a UFLAg-GFP::PEP $\mathrm{Ag}_{\mathrm{Ag}}$ cell line

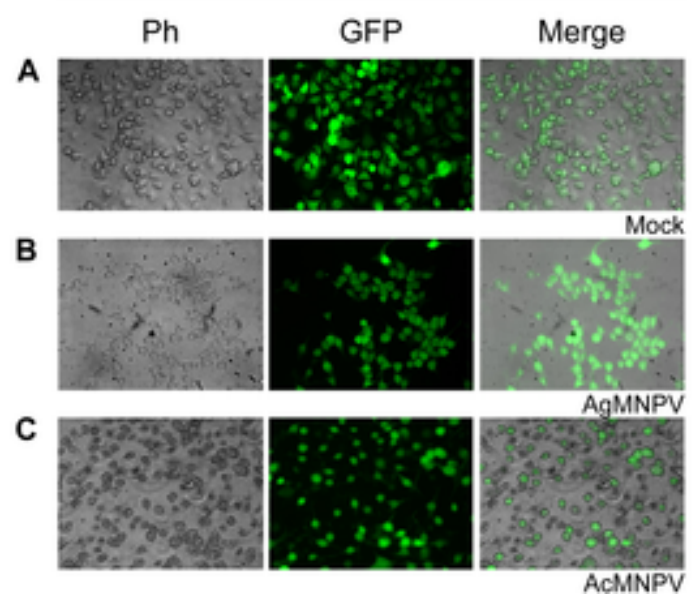

of the fusion GFP::PEP $\mathrm{Ag}_{\mathrm{Ag}}$ in the OBs (Fig. 3b). The incorporation efficiency of GFP::PEP ${ }_{\mathrm{Ag}}$ using UFLAg-GFP:: $\mathrm{PEP}_{\mathrm{Ag}}$ cells was analyzed in AgMNPV and AcMNPV OBs. Parallel cultures of this insect cell line were infected with either virus at the same moi, in duplicate and, purified OBs were counted by fluorescent microscopy in triplicate. The percentages of fluorescent OBs (GFP-positive) versus non fluorescent OBs are shown in a bar graph (Figs. 3c and S1). Student's $t$ test showed no significant differences between the two analyzed baculoviruses. Scanning electron microscopy (SEM) analysis showed a smooth surface typical of intact polyhedra for both viruses (Fig. 3d). SEM images used to estimate the mean diameter of the polyhedra resulted in $3 \mu \mathrm{m}$ and $230 \mu \mathrm{m}$ for AgMNPV and AcMNPV OBs, respectively. This measurements agree with previously reported data (Coulibaly et al., 2009; Pombo et al., 1998). In summary, these results indicated a good performance of a stably transformed insect cell line to supply the recombinant protein GFP::PEP $\mathrm{Ag}_{\mathrm{A}}$ and deliver it to the wild type baculovirus OBs without disrupting the viral envelope.

\subsection{OBs mass spectrometry analysis}

OBs obtained upon infection of UFLAg-GFP::PEP Ag $_{\text {with AgMNPV or }}$ AcMNPV, in parallel experiments, were analyzed by tandem mass spectrometry to characterize their protein composition and to confirm the incorporation of GFP:: $\mathrm{PEP}_{\mathrm{Ag}}$ protein. LC-MS/MS profiles were similar to those reported by others for wild type AcMNPV and AgMNPV OBs (Braconi et al., 2014; Wang et al., 2010): a total number of 78 and 66 proteins were identified in AcMNPV and AgMNPV packaged OBs, respectively (Table S1). Ten and seven GFP:: $\mathrm{PEP}_{\mathrm{Ag}}$ unique peptides were identified in AgMNPV and AcMNPV OBs, respectively, confirming the presence of the recombinant fusion protein in the OBs (Fig. 4a). Most importantly, in the case of AcMNPV endogenous PEP could also be identified confirming that the competition between both active PEPs were not mutually exclusive. In addition, a label-free quantification method based on spectral counting was used in LC-MS/MS. Normalized emPAI values obtained by Proteome Discoverer ${ }^{\mathrm{TM}}$ search software were used as a rough estimation of the amount of GFP:: $\mathrm{PEP}_{\mathrm{Ag}}$ in AcMNPV or AgMNPV OBs (Table S2) (Ishihama et al., 2005; Shinoda et al., 2009). For the GFP::PEP $\mathrm{Ag}_{\mathrm{Ag}}$ protein in AgMNPV OB and AcMNPV OB samples the molar percentages were 8.82E-02 and 1.1E-04, respectively. This approach reflected that the AgMNPV OBs incorporated approximately a hundred times more chimeric protein than the AcMNPV OBs. Further analyses are needed to make a more accurate quantification of the fusion GFP::PEP $\mathrm{Ag}_{\mathrm{A}}$ in the OBs.

\section{b \\ UFLAg-GFP cell line}

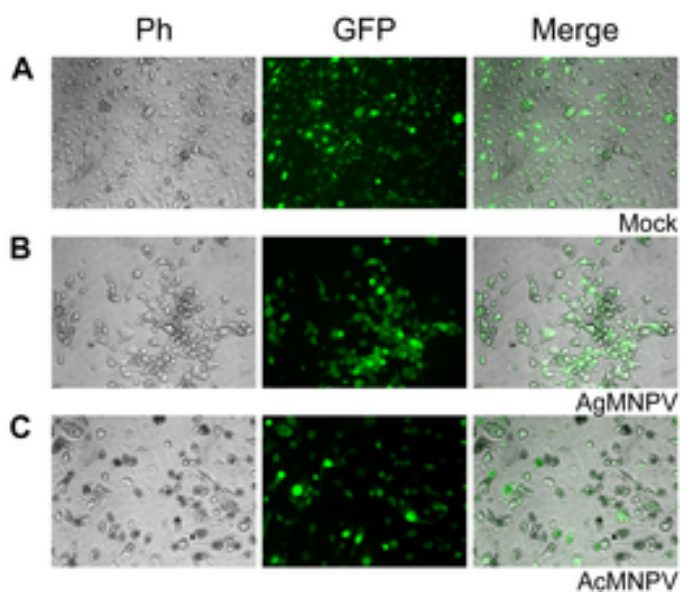

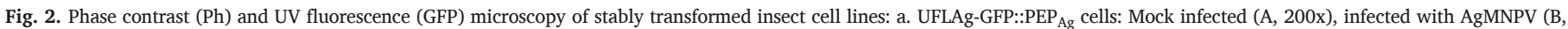
$200 \times$ ), AcMNPV (C, $200 \times$ ) 3 d p.i. b. UFLAg-GFP cells: Mock infected (A, 200x), infected with AgMNPV (B, 200x), AcMNPV (C, 200×) 3 dp.i. 
a

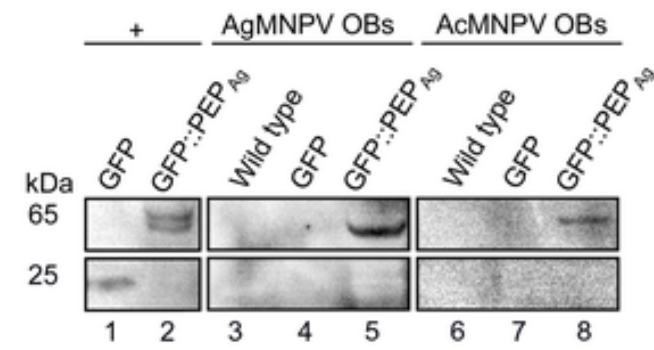

C

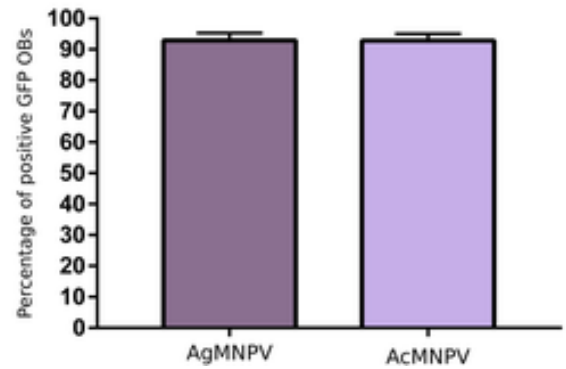

b
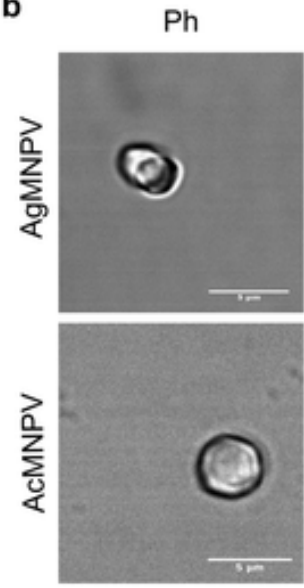

d AgMNPV

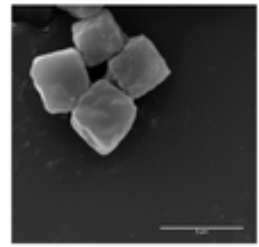

AcMNPV
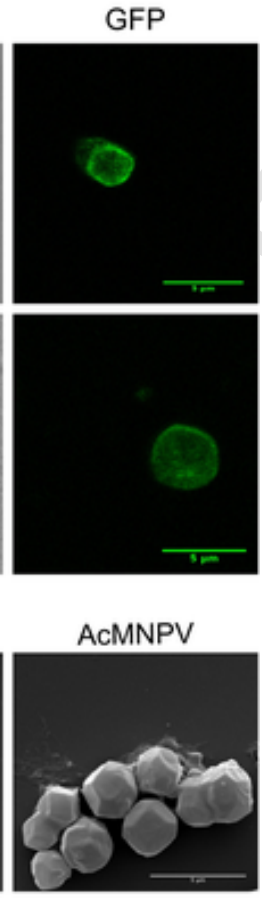

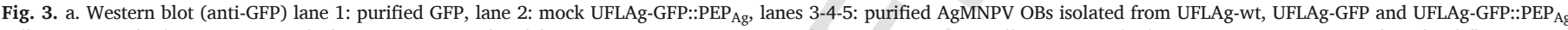

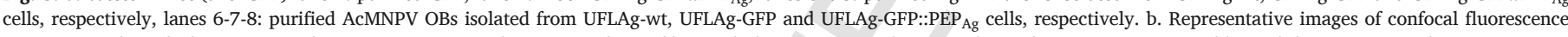

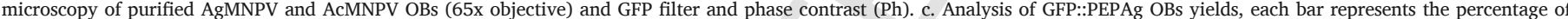

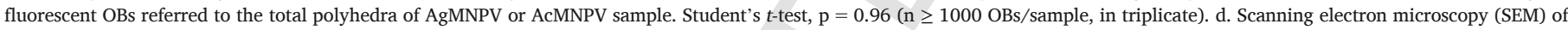
purified OBs from AgMNPV and AcMNPV $(10000 \times)$.
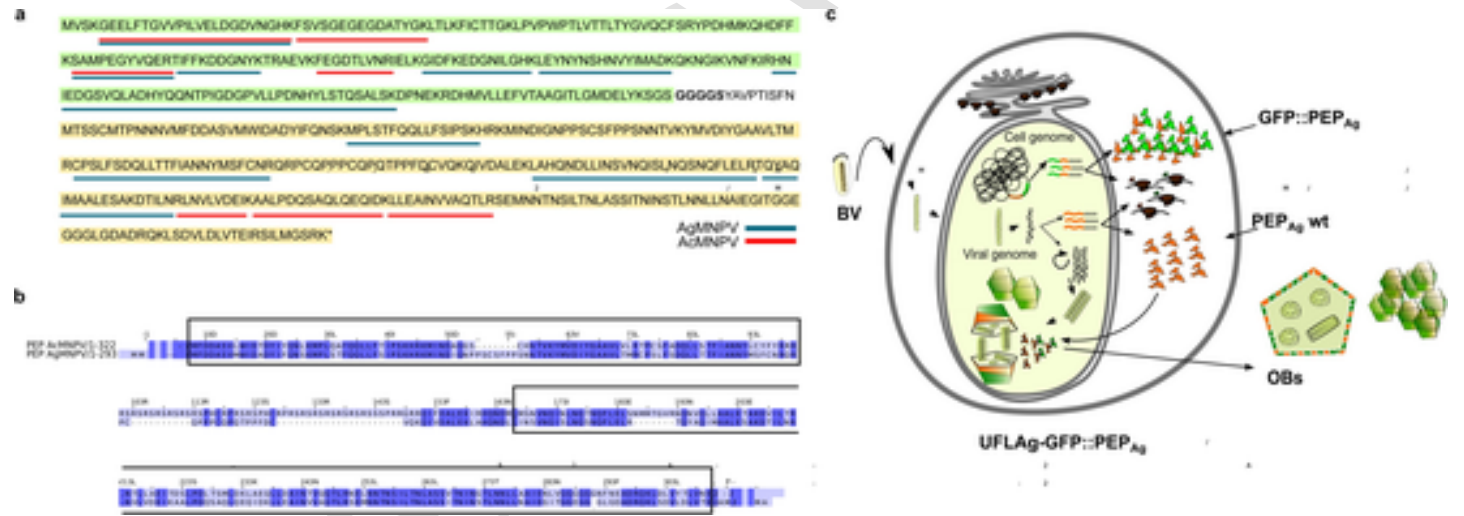

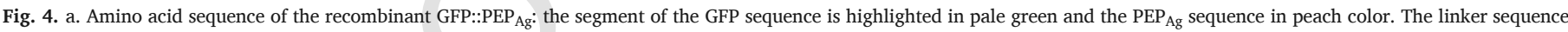

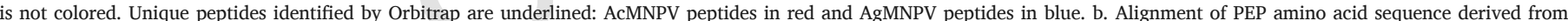

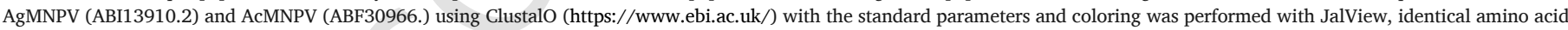

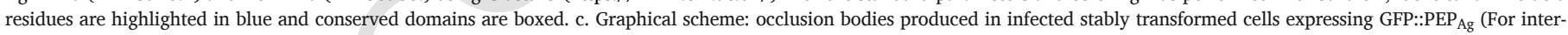
pretation of the references to colour in this figure legend, the reader is referred to the web version of this article).

\section{Discussion}

Formulation of biopesticides based on wild type baculoviruses has been widely tested and approved for field use by regulatory authorities in many countries. Even though recombinant baculoviruses were reported to increase the speed of kill or reduce the lethal doses needed to control of the target pest (Kroemer et al., 2015; López et al., 2018,; Yang et al., 2017), extensive efforts should be made to determine the environmental impact (Haase et al., 2015; Hoy, 2019; Tiedje et al., 2014). Regulations preventing the release of genetically modified viruses on a broad scale encouraged us to develop a proof of concept to test the ability of a transformed cell line, UFLAg-GFP::PEP $\mathrm{Ag}_{\mathrm{Ag}}$ to express and deliver the fusion polypeptide GFP::PEP $\mathrm{Ag}$ to different wild type OBs (Fig. 4c). In this study we used wild type AgMNPV and AcMNPV, two group I alphabaculoviruses with a narrow and a broad host range, respectively. The morphology of these decorated OBs showed that both AgMNPV and AcMNPV decorated with GFP::PEP $\mathrm{Ag}_{\mathrm{Ag}}$ had a normal shape and size, in accordance with previous reports for wild type OBs (Sajjan and Hinchigeri, 2016; Wang et al., 2010). Even though PEP is a virus species-specific protein, AcMNPV encoded PEP is expressed and assembled to form the envelope and allows interactions with the heterologous GFP:: $\mathrm{PEP}_{\mathrm{Ag}}$ constitutively expressed in engineered

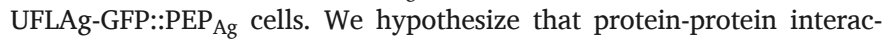
tions between different baculoviral PEPs are highly dependent on the sequence similarity and the evolutionary distance between them. This observation is supported by the lack of complementation between AcM- 
NPV PEP and orthologs of distantly related viruses, like HearNPV and CpGV with $41 \%$, $24.8 \%$ sequence identity, respectively (Li et al., 2015). In contrast, amino acid sequence alignment between AgMNPV and AcMNPV PEP showed an overall $63 \%$ identity (Fig. 4). Further studies are needed to determine the composition of the polyhedron envelope and the interaction of PEP within this structure. The proteomic analysis yielded OB protein profiles which are in accordance with those reported in the literature pointing at an unaltered composition of nucleocapsid and ODV inside the OBs. The detection of GFP:: $\mathrm{PEP}_{\mathrm{Ag}}$ in both baculovirus OBs indicated that our strategy was successful in delivering a recombinant protein encoded in trans by a transformed UFLAg-GFP::PEP ${ }_{A g}$ cell line. Regarding the label-free quantification, the normalized emPAI showed a lower incorporation of the chimeric protein by AcMNPV OBs, which is consistent with the reported characteristic of pep gene product as virus-species-specific (Li et al., 2015). For future label-free proteomic quantification experiments saturation with highly expressed proteins such as POLH should be minimized. We presented a novel strategy to incorporate foreign proteins into wild type baculovirus occlusion bodies avoiding the need to genetically modify the viruses for their use as bioinsecticide. Since polyhedron envelope is the first structure that must be disassembled to allow the release of virions (ODV) in the midgut, the incorporation of insecticidal proteins into this structure would contribute to a faster invasion of intestinal epithelium of target insect larvae. Proteins like v-cath, chitinase, metalloproteinases would be interesting choices. On the other hand, it was shown that lectins have the ability to deliver proteins from the midgut to the nervous system of the larvae (Fitches et al., 2012). Lectins should be exploited to generate fusion peptides with arthropod-specific toxins that have the potential to improve their insecticidal properties. This work is aimed at exploring the transformed insect cell lines as a new biotechnological platform that allows the generation of decorated occlusion bodies.

Packaged OBs maintain a wild type genome and exhibit a homogeneous size distribution comparable to the viruses propagated in non-transformed cells. No integration of host genomic DNA sequences has been detected by PCR in BVs, or after passaging the viral progeny from UFLAg-GFP::PEP ${ }_{A g}$ cells in UFLAg cells. Even though the occurrence of a recombination event between the cellular and baculoviral genome had been reported in the past (Mangor et al., 2001) so far we have never detected integration of host DNA sequences in the baculoviral genome (data not shown). The conditions that allowed Mangor et al. to detect gp64 knock out AcMNPV (vAc ${ }^{64-}$, unable to infect and propagate $S f 9$ cells) that acquired VSV G sequences from Sf ${ }^{\text {VSV-G }}$ cell line expressing this glycoprotein were quite different from those described in our study: i.e., $\mathrm{vAc}^{64-}$ was passaged many times in $\mathrm{Sf} 9^{\mathrm{VSV}-\mathrm{G}}$; the VSV $\mathrm{G}$ sequences in the cell genome were flanked by extensive baculovirus sequences from the $p 10$ locus that maximized the probability of homologous recombination with the $\mathrm{vAc}^{64-}$ genome, thus, generating a virus with the selective advantage of infecting, replicating and propagating independently in Sf9 cells (as opposed to $\mathrm{vAc}^{64-}$ ); our wt viruses are fit for propagation, the chances of acquiring a second copy of a chimeric pep gene (in addition to the wt pep gene) are minimized by the lack of extensive flanking viral sequences; in addition, such recombinant virus would not exhibit a selective advantage. Therefore, even though we cannot absolutely rule out the chance of integration of host DNA fragments into the baculoviral genome, this is a very unlikely possibility in a single amplification of the AcMNPV and AgMNPV in UFLAg-GFP::PEP $P_{A g}$ cells. Nevertheless, for future applications of this system exhaustive analysis should be performed to rule out any genome rearrangements.

The incorporation of exogenous proteins expressed by the cell line brings a new perspective to the use of OBs as carriers for biotechnological uses, including applications other than baculovirus-based biopesticides, such as OB-associated antigens and the use of polyhedra as slow delivery of bioactive polypeptides (López et al., 2018,López et al., 2018; Fabre et al., 2019).

\section{Funding}

This work was supported by Fondo para la Investigación Científica y Tecnológica (FONCyT), Agencia Nacional de Promoción Científica y Tecnológica (ANPCyT), PICT 2014-1827, PICT 2016-2022; Universidad Nacional de La Plata.

\section{Ethical approval}

This article does not contain any studies with human participants or animals performed by any of the authors.

\section{Declaration of Competing Interest}

The authors declare that they have no known competing financial interests or personal relationships that could have appeared to influence the work reported in this paper.

\section{Acknowledgments}

The authors are grateful to Larisa Vasquez, Paula Giménez and Silvana Tongiani (Instituto de Biotecnología y Biología Molecular, IBBM) for their technical assistance.

\section{Appendix A. Supplementary data}

Supplementary material related to this article can be found, in the online version, at doi:https://doi.org/10.1016/j.jbiotec.2019.10.017.

\section{References}

Ali, M.P., Kato, T., Park, E.Y., 2015. Improved insecticidal activity of a recombinant baculovirus expressing spider venom cyto-insectotoxin. Appl. Microbiol. Biotechnol. 99, 10261-10269. doi:10.1007/s00253-015-6846-0.

Braconi, C.T., Ardisson-Araujo, D.M., Leme, A.F., Oliveira, J.V., Pauletti, B.A., Garcia-Maruniak, A., Ribeiro, B.M., Maruniak, J.E., Zanotto, P.M., 2014. Proteomic analyses of baculovirus Anticarsia gemmatalis multiple nucleopolyhedrovirus budded and occluded virus. J. Gen. Virol. 95 (4), 989. doi:10.1099/vir.0.061127-0.

Bradford, M.M., 1976. A rapid and sensitive method for the quantitation of microgram quatities of protein utilizing the principle of protein-dye binding. Anal. Biochem. 72, 248-254. doi:10.1016/0003-2697(76)90527-3.

Chen, L., Xiang, X., Yang, R., Hu, X., Cao, C., Malik, F.A., Wu, X., 2013. Immobilization of foreign protein in BmNPV polyhedra by fusion expression with partial polyhedrin fragments. J. Virol. Methods 194, 185-189. doi:10.1016/j.jviromet.2013.08.020.

Coulibaly, F., Chiu, E., Gutmann, S., Rajendran, C., Haebel, P.W., Ikeda, K., Mori, H. Ward, V.K., Schulze-Briese, C., Metcalf, P., 2009. The atomic structure of baculovirus polyhedra reveals the independent emergence of infectious crystals in DNA and RNA viruses. Proc. Natl. Acad. Sci. U. S. A. 106, 22205-22210. doi:10.1073/ pnas.0910686106.

Fabre, M.L., Arrías, P., Masson, T., Pidre, M.L., Romanowski, V., 2019. Baculovirus-derived vectors for immunization and therapeutic applications. In: Ennaji, M.M. (Ed.), Emerging and Reemerging Viral Pathogens, 1st ed. Elsevier-Academic Press. [ISBN: 9780128149669].

Fitches, E.C., Pyati, P., King, G.F., Gatehouse, J.A., 2012. Fusion to snowdrop lectin magnifies the oral activity of insecticidal $\omega$-Hexatoxin-Hv1a peptide by enabling its delivery to the central nervous system. PLoS One 7 (6), e39389. doi:10.1371/ journal.pone.0039389.

Haase, S., Ferrelli, M.L., Pidre, M.L., Romanowski, V., 2013. Genetic engineering of baculoviruses. In: Romanowski, V. (Ed.), Current Issues in Molecular Virology - Viral Genetics and Biotechnological Applications. InTech. ISBN: 978-953-51-1207-5. doi:10.5772/56976.

Haase, S., Sciocco-Cap, A., Romanowski, V., 2015. Baculovirus insecticides in Latin America: historical overview, current status and future perspectives. Viruses 7, 2230-2267. doi:10.3390/v7052230.

Harrison, R.L., Herniou, E.A., Jehle, J.A., Theilmann, D.A., Burand, J.P., Becnel, J.J., Krell, P.J., van Oers, M.M., Mowery, J.D., Bauchan, G.R., Consortium, I.R., 2018. ICTV virus taxonomy profile: Baculoviridae. J. Gen. Virol. 99, 1185-1186. doi:10.1099/ jgv.0.001107.

Hegedus, D., Erlandson, M., Gillott, C., Toprak, U., 2009. New insights into peritrophic matrix synthesis, architecture, and function. Annu. Rev. Entomol. 54, 285-302. doi:10.1146/annurev.ento.54.110807.090559.

Hoy, M.A., 2019. Genetic modification of pest and beneficial insects for pest management programs. Insect Mol. Genet. 563-620. [ISBN: 9780128152300]. doi:10.1016/ B978-0-12-815230-0.00014-5.

Ishihama, Y., Oda, Y., Tabata, T., Sato, T., Nagasu, T., Rappsilber, J., Mann, M., 2005. Index (emPAI) for estimation of absolute protein amount in proteomics by the number of sequenced peptides per protein. Mol. Cell Proteom. 1265-1272. doi:10.1074/ mcp.M500061-MCP200. 
Jehle, J.A., Blissard, G.W., Bonning, B.C., Cory, J.S., Herniou, E.A., Rohrmann, G.F., Theilmann, D.A., Thiem, S.M., Vlak, J.M., 2006. On the classification and nomenclature of baculoviruses: a proposal for revision. Arch. Virol. 151, 1257-1266. doi:10.1007/ s00705-006-0763-6.

Kroemer, J.A., Kroemer, J.A., Bonning, B.C., Harrison, R.L., 2015. Expression, delivery and function of insecticidal proteins expressed by recombinant baculoviruses. Viruses 7 , 422-455. doi:10.3390/v7010422.

Levy, S.M., Falleiros, Â., Moscardi, F., Gregório, E.A., 2011. The role of peritrophic membrane in the resistance of Anticarsia gemmatalis larvae (Lepidoptera: Noctuidae) during the infection by its nucleopolyhedrovirus (AgMNPV). Arthropod Struct. Dev. 40, 429-434. doi:10.1016/j.asd.2011.05.003.

Li, J., Zhou, Y., Lei, C., Fang, W., Sun, X., 2015. Improvement in the UV resistance of baculoviruses by displaying nano-zinc oxide-binding peptides on the surfaces of their occlusion bodies. Appl. Microbiol. Biotechnol. 99, 6841-6853. doi:10.1007/ s00253-015-6581-6.

López, M.G., Diez, M., Alfonso, V., Taboga, O., 2018a. Biotechnological applications of occlusion bodies of Baculoviruses. Appl. Microbiol. Biotechnol. 10. doi:10.1007/ s00253-018-9130-2.

López, M.G., Pallarés, H.M., Alfonso, V., Carmona, S.J., Farber, M., Taboga, O., Wilkowsky, S.E., 2018b. Novel biotechnological platform based on baculovirus occlusion bodies carrying Babesia bovis small antigenic peptides for the design of a diagnostic enzyme-linked immunosorbent assay (ELISA). Appl. Microbiol. Biotechnol. 102, 885-896. doi:10.1007/s00253-017-8662-1.

Lynn, D.E., Harrison, R.L., 2016. Available lepidopteran insect cell lines. In: Methods in Molecular Biology. doi:10.1007/978-1-4939-3043-2_6.

Mangor, J.T., Monsma, S.A., Johnson, M.C., Blissard, G.W., 2001. A GP64-null baculovirus pseudotyped with vesicular stomatitis virus g protein. J. Virol. 75 (6), 2544-2556. doi:10.1128/jvi.75.6.2544-2556.2001.

Masson, T., Fabre, M.L., Ferrelli, M.L., Pidre, M.L., Romanowski, V., 2019. Protein composition of the occlusion bodies of Epinotia aporema granulovirus. PLoS One 14, e0207735. doi:10.1371/journal.pone.0207735.

Mitsuhashi, W., Kawakita, H., Murakami, R., Takemoto, Y., Saiki, T., Miyamoto, K., Wada, S., 2007. Spindles of an entomopoxvirus facilitate its infection of the host in- sect by disrupting the peritrophic membrane. J. Virol. 81, 4235-4243. doi:10.1128/ JVI.02300-06.

Moscardi, F., 1999. Assessment of the application of baculoviruses for control of Lepidoptera. Annu. Rev. Entomol. 44, 257-289. doi:10.1146/annurev.ento.44.1.257.

O'Reilly, D.R., Miller, L.K., Luckow, V.A., 1994. Baculovirus Expression Vectors: A Laboratory Manual. Oxford University Press.

Ogay, I.D., Lihoradova, O.A., Azimova, S.S., Abdukarimov, A.A., Slack, J.M., Lynn, D.E., 2006. Transfection of insect cell lines using polyethylenimine. Cytotechnology 51, 89-98. doi:10.1007/s10616-006-9022-7.

Pombo, V., Velloso, L.M., Ribeiro, B.M., Báo, S.N., 1998. Structural and ultrastructural changes during the infection of UFL-AG-286 cells with the baculovirus AgMNPV. J. Invertebr. Pathol. 72, 239-245. doi:10.1006/jipa.1998.4788.

Rohrmann, G.F., 2013. Baculovirus Molecular Biology. 3rd ed. Bethesda Natl. Cent. Biotechnol. Inf. (US). https://www.ncbi.nlm.nih.gov/pubmed/24479205.

Sajjan, D.B., Hinchigeri, S.B., 2016. Structural organization of baculovirus occlusion bodies and protective role of multilayered polyhedron envelope protein. Food Environ. Virol. 8 (1), 100. doi:10.1007/s12560-016-9227-7.

Shinoda, K., Tomita, M., Ishihama, Y., 2009. emPAI Calc-for the estimation of protein abundance from large-scale identification data by liquid chromatography-tandem mass spectrometry. Bioinformatics 26 (4), 576-577. doi:10.1093/bioinformatics/ btp700.

Sieburth, P., Maruniak, J., 1988. Susceptibility of an established cell line of (Lepidoptera: Noctuidae) to three nuclear polyhedrosis viruses. J. Invertebr. Pathol. 52, 453-458. doi:10.1016/0022-2011(88)90058-4.

Tiedje, J.M., Colwell, R.K., Grossman, Y.L., Hodson, R.E., Lenski, E., Mack, R.N., Regal, P.J., Ecology, S., Apr, N., 2014. The planned introduction of genetically engineered or ganisms: ecological considerations and recommendations. Ecological Society of America 70, 298-315.

Wang, R.R., Deng, F., Hou, D.H., Zhao, Y., Guo, L., Wang, H.L., Hu, Z.H., 2010. Proteomics of the Autographa californica nucleopolyhedrovirus budded virions. J. Virol. 84, 7233-7242. doi:10.1128/jvi.00040-10.

Yang, S., Zhao, L., Ma, R., Fang, W., Hu, J., Lei, C., Sun, X., 2017. Improving baculovirus infectivity by efficiently embedding enhancing factors into occlusion bodies. Appl. Environ. Microbiol. 83 (14), 595-607. AEM.00595-17. doi:10.1128/AFM.00595-17. 